Main themes around hospital admissions were emergency ambulance requests by care homes (33\%), arranged admissions by GPs $(21 \%)$, and family request $(14 \%)$. Variation was found in the way care homes and GPs managed frail and declining patients, and residents were sometimes admitted to hospital against the wishes of care home staff and families. Other issues identified were difficulties in accessing services, lack of reflection, and conflicting roles.

Conclusions Service provision was fragmented. The author suggests that GPs, Intermediate Care, Ambulance Services, GP Out of Hours, palliative care teams and care homes work together closely to address the issues. Also suggested are regional groups to co-ordinate and monitor end of life care in care homes. National accreditation should be introduced for end of life care in care homes, and regulatory bodies (such as Care Inspectorate Wales) should inspect care homes accordingly.

This research fills a gap in our knowledge, and its findings can be applied internationally.

\section{A PROJECT TO EXPLORE THE POTENTIAL USE OF DIGITAL TECHNOLOGY TO AID HOSPICE CARE; FOCUSSING ON REMOTE CONSULTING}

Jennifer Palfrey, Craig Gannon. Princess Alice Hospice

\subsection{6/bmjspcare-2019-ASP.111}

Background Princess Alice Hospice's current strategy promotes exploring different ways of working to increase end-of-life care and support, in more sustainable ways. This includes the potential to integrate digital technology into hospice care. This project aimed to assess the willingness and capability of patients and families, and healthcare professionals to engage with new technologies for consultations in an end-of-life care setting. It sought to identify opportunities for improved, efficient communication and highlight any potential barriers to the use of telemedicine.

Method A mixed methods approach was used, first focusing on established technology users in order to understand their needs and preferences before expanding to users who were less digitally connected. Data was collected in: questionnaires, feedback and collaboration workshops with patients/carers, internal and external professionals, trial sessions of telemedicine. A thematic analysis was then conducted.

Results

- Greater than 66\% participants had access to internet enabled devices

- $80 \%$ people use the internet on a daily basis

- $63 \%$ participants used Skype or equivalent before; $47 \%$ of these were using it either daily or weekly.

'[I'm] very comfortable using video calls. I would happily swap some visits for a video call on Skype or FaceTime.'

'[Using video calls] would be fine if I felt comfortable with the technology and I knew the person I was talking to and already had a relationship with them.'

Conclusions Telemedicine is acceptable to many people who use the hospice services. Indeed, patients described telemedicine as having some logistical advantages over home visits. However, 1st consultations should continue to be face-to-face in order to establish a relationship, do an environmental assessment and consider whether telemedicine would be appropriate for future contacts.

\section{SPECIALIST PALLIATIVE CARE CNS SUPPORT SERVICE FOR CARE HOMES IN SOUTH TEES - DEVELOPMENT AND REVIEW}

Trish Sealy, Julie Tuttle, Sue Gent, Rachel Fawcett. South Tees NHS FT

\subsection{6/bmjspcare-2019-ASP.112}

Background Middlesbrough Redcar Cleveland has a population of approximately 300000 with $>130$ registered care homes. MRC Community SPCT receives around 1300 referrals/year of which around $8 \%$ come directly from care homes. Across the locality in 2017/8 22.9\% of deaths occurred in care homes. An existing single-handed CNS for care homes had identified areas for development but was unable to progress this due to limited capacity.

In 2016, funding accessed via the Better Care Fund secured an additional Specialist Palliative Care CNS within the Community SPCT to support the existing post-holder and with a remit to extend and develop training and support within the Care Home setting, with particular emphasis on verification of expected death, syringe driver training and advance care planning.

Results Between January and August 2018 an education programme delivered syringe driver updates to $>100$ staff, and verification of death training to $>80$ staff. A level 1 programme (consisting of a single broad-reaching introductory education session) for support staff has been introduced and rolled out tomore than 40 care homes. Pre and post-course analysis shows significant improvements in knowledge levels and confidence.

In $2017 / 1817 \%$ of patients dying whilst on the caseload of the community SPCT died in a care home. Between April 2018 and September 2018 this had risen to $32 \%$.

Nurse verification of death within the care homes represents a shift in work which was previously undertaken by the OOH service.

Direct advance care planning including discussion and implementation of treatment escalation and DNACPR orders, and provision of anticipatory end of life medications for symptom management has taken place in $>40$ individuals.

Conclusions Targeted interventions within care home settings can empower staff and improve outcomes in care at the end of life.

\section{EXPLORING CLINICIAN ATTITUDES TO RESPECT AT CHESTERFIELD ROYAL HOSPITAL}

Vanessa Jackson, David Brooks, Gemma Cort, Joanne Froud. Chesterfield Royal Hospital

\subsection{6/bmjspcare-2019-ASP.113}

Background ReSPECT is a joint venture between professional bodies in the UK led by the Resuscitation Council to standardise documentation of DNAR decisions and patients' preferences for care. ReSPECT was introduced in Chesterfield Royal Hospital (CRH), Derbyshire in June 2018.

Aims The aims of this project were: 1) Evaluate hospital clinicians' views on ReSPECT and 2) Highlight which aspects of the form clinicians are finding difficult. This information should help to improve the ReSPECT process and guide future training.

Methods We conducted a survey of ten questions on Survey Monkey, collecting a mix of qualitative and 
quantitative data. This was distributed via email to all doctors at CRH in September 2018 three months after the implementation date. Results were analysed and distributed to stakeholders.

Results A total of 43 doctors completed the survey, representing a range of grades and specialties. Mean score for how user-friendly respondents found the form was 6 out of 10 . The proportion of respondents who found ReSPECT useful in planning patient care was 58\%. However, approximately three quarters of respondents had encountered difficulties with the form $(74 \%)$.

Six broad themes around difficulties with ReSPECT were identified: 1) Time required, 2) CPR decision, 3) Complexity, 4) Scale - life sustaining vs comfort, 5) Paper format and storage issues and 6) Capacity assessment. Suggestions for improvement of the ReSPECT process included: larger boxes on the form, clearer emphasis on CPR decision, and improving systems for handover between care settings.

Conclusion The ReSPECT process has implications for the palliative care of patients admitted to hospital. The results of this survey demonstrate concerns from clinicians, and suggestions for improvement. The suggestions of CRH staff could provide a basis for guidance for other implementers and improvements to future versions of the form nationally.

\section{REDESIGNING SPECIALIST PALLIATIVE AND HOSPICE CARE TO MEET THE NEEDS OF PEOPLE WITH FRAILTY}

Caroline Nicholson, Rob George, Victor Pace, Heather Richardson. St Christopher's Hospice, King's College London

\subsection{6/bmjspcare-2019-ASP.114}

Background Older people with multimorbidities will dominate palliative care in coming decades. For them, dying can be slow and unpredictable. Care models integrated across the spectrum of geriatric and palliative medicine, health and social care, are emerging. We draw on current theory and evidence to develop a framework that assists current services attune to the needs of this population.

Methods We used stepped framework construction.Eight clinicians and service users reviewed and refined initial findings.

- to scope the palliative/geriatric literature and define focus, inclusion and exclusion criteria for a proactive palliative care model (Seymour 2014). Documents from 102 research, review, policy papers and grey literature were included.

- Face-validity testing extended to a wider consultation across care sectors, care commissioners and providers.

Findings Built around emergent concerns of recipient older people and their family as care partners, the framework identifies three intervention levels that work

- differently as clinicians;

- in partnership to improve services and the broader care system;

- $\quad$ as support to wider societal change.

An optimal clinical response requires:

- Meticulous, systematic needs assessment using the right tools;

- Parallel planning across specialties for several possible outcomes;
- Sensitive discussions that are realistic about health conditions and age;

- Access to advanced palliative practice competent to stop as well as start treatments, and work across relevant specialties including rapid response in the community;

- Access to rehabilitative care that optimizes function;

- 'Watchful Waiting', where purposeful, low-level proactive engagement with people and families identifies incremental changes and responds appropriately;

- Effective, relevant bereavement care.

Outcome This Age Attuned Hospice framework offers a flexible structure for organisations seeking to become more responsive to the older, frail population whilst maintaining effective current specialist practice.

\section{INTRODUCING THE TRAJECTORY TOUCHPOINT TECHNIQUE: A SYSTEMATIC METHODOLOGY FOR CAPTURING THE VOICES OF PALLIATIVE CARE SERVICE USERS}

Lynn Sudbury-Riley, Philippa Hunter-Jones, Ahmed Al-Abdin. University of Liverpool

\subsection{6/bmjspcare-2019-ASP.115}

Background We present the trajectory touchpoint technique (TTT), a new methodology to appreciate all user experiences of palliative care. Better understanding of user needs means providers can become more patient-centered. However, no available tools capture experience of all aspects of palliative care. Consequently we developed the new methodology, allowing a deep dive into the palliative care journeys of patients and their families.

Methods Using concepts from the social sciences, we blended service blueprinting, customer journeys, touchpoints, servicescapes, experienced based co-design, and rich pictures methodology; producing a technique that systematically captures palliative care user experiences from seven major dimensions. The technique incorporates all aspects of palliative care that are important to the user, integrating emotional, physical, sensorial, and social elements of their experience. We have tested and refined the TTT in a collaboration with hospices and palliative care providers $(n=8)$, successfully capturing the voices $(n=223)$ of palliative care patients and their families.

Results The TTT overcomes many data collection difficulties typical to palliative care. The TTT is easy to understand and use, yet costs a fraction of alternative techniques while allowing for a broader perspective into patient-centeredness, incorporating both the clinical encounter and macro-level organizational structures and processes. Importantly, results demonstrate the TTT's ability to uncover areas for improvement that alternative methodologies have failed to identify, despite many of our collaborating organizations already being rated as 'outstanding'.

Conclusions Data collection methods that reveal actionable results which lead to meaningful changes are crucial to implementing improvements to palliative care. Our TTT responds to this need. Based on our results, our collaborating organizations have already begun to make improvements to the ways in which palliative care services are designed, delivered, communicated, co-created, and connected to the wider health care ecosystem. 